\title{
ALGEBRAS GENERATED BY ODD DERIVATIONS
}

\section{CLAUDE ROGER}

Communicated by Guowu Meng

\begin{abstract}
Given an associative supercommutative algebra equipped with an odd derivation, one considers the space of vector fields it defines, and show, under suitable hypothesis, they form a Jordan superalgebra; in contrast with the Lie superalgebras of Virasoro type constructed from even derivations. Relations with Anti Lie algebras studied by Ovsienko and collaborators are then shown.
\end{abstract}

MSC: 17B60,17B65,17B68, 81Q60

Keywords: Superalgebras, supergeometry

Let $\mathcal{A}$ be an $\mathbb{Z} / 2 \mathbb{Z}$-graded, associative and graded commutative algebra.The degree of an element $a \in \mathcal{A}$ will be denoted by $|a|$, the same for the degree of an application; we shall consider derivations of $\mathcal{A}$. A map $\delta: \mathcal{A} \rightarrow \mathcal{A}$ is a derivation if for any $a, b \in \mathcal{A}$, one has

$$
\delta(a b)=\delta(a) b+(-1)^{|a||\delta|} a \delta(b) .
$$

It is well-known that the space of derivations of a commutative associative algebra is a Lie algebra through commutator, a generic example being derivations of the algebra of smooth functions on a differentiable manifold, isomorphic to the Lie algebra of tangent vector fields through Lie derivative. This fact generalizes to the $\mathbb{Z} / 2 \mathbb{Z}$-graded case: graded bracket of derivations induces a Lie superalgebra structure, as can easily be deduced from the formula

$$
\left[\delta_{1}, \delta_{2}\right]=\delta_{1} \circ \delta_{2}-(-1)^{\left|\delta_{1}\right|\left|\delta_{2}\right|} \delta_{2} \circ \delta_{1} .
$$

For basic definitions and results about superalgebra, see [2, vol.1, Part 1].

\section{About Virasorisation}

We shall generalize here the construction of Virasoro algebra from the commutative and associative algebra of smooth functions on the unit circle with its natural derivative (cf. [4] for basic results about Virasoro algebra). Let $\delta$ be a derivation of $\mathcal{A}$, and $a \in \mathcal{A}$, then $a \delta$ defined as $[a \delta](b)=a \delta(b)$, is a derivation of degree 
$|a \delta|=|a|+|\delta|$; so if $\delta$ is even, then $|a \delta|=|a|$. One can then define the graded commutator of two such derivations as

$$
[a \delta, b \delta]=\left(a \delta b-(-1)^{|a||b|} b \delta a\right) \delta .
$$

One obtains a Lie superalgebra denoted by $\mathcal{V}(\mathcal{A})$ which can be called virasorisation of $\mathcal{A}$, and the parity satisfies: $\mathcal{V}(\mathcal{A})_{i}$ is isomorphic to $\mathcal{A}_{i}$ for $i=0,1$ modulo 2. One thus recover Virasoro algebra together with some of its supersymmetric partners, such as superconformal algebras like Vect $(1 \mid 1)$, but that construction is far more general (cf. [8], Appendix).

\section{When the Derivation is Odd}

In that case, we can define a graded space $\mathcal{V}(\mathcal{A})$, generated by the $a \delta$, and $\mathcal{V}(\mathcal{A})_{i}$ is isomorphic to $\mathcal{A}_{i+1}$ for $i=0,1$ modulo 2 .

The choice of signs in the bracket of $\mathcal{V}(\mathcal{A})$ is imposed by parity. Elementary computations shows that in order to cancel all terms in $\delta^{2}$ (in physicist's language, the algebra must "close"), one must set

$$
[a \delta, b \delta]=\left(a \delta b+(-1)^{(|a|+1)(|b|+1)} b \delta a\right) \delta .
$$

The bracket is then a commutator if $|a|=|b|=0$, an anticommutator in all other cases, one has to study the kind of algebraic structures we just obtained.

$$
\begin{aligned}
& \mathcal{V}(\mathcal{A})_{0} \times \mathcal{V}(\mathcal{A})_{0} \rightarrow \mathcal{V}(\mathcal{A})_{0} \\
& \mathcal{V}(\mathcal{A})_{0} \times \mathcal{V}(\mathcal{A})_{1} \rightarrow \mathcal{V}(\mathcal{A})_{1} \\
& \mathcal{V}(\mathcal{A})_{1} \times \mathcal{V}(\mathcal{A})_{1} \rightarrow \mathcal{V}(\mathcal{A})_{0}
\end{aligned}
$$

One can check immediately that those multiplications satisfy the same symmetryantisymmetry conditions as the Lie antialgebras defined and studied by Valentin Ovsienko in [7].

Let us first consider an important particular case, when $\mathcal{A}$ is the algebra of functions on the supercircle $S^{1 \mid 1}$ in variables $t, \theta$, and when $\delta=D_{\theta}=\theta \frac{\partial}{\partial t}-\frac{\partial}{\partial \theta}$ (remember the relation $D_{\theta} \circ D_{\theta}=\frac{\partial}{\partial t}$ ). This algebra will be denoted $\mathcal{V}(\mathcal{A}(1))$. One has then

Proposition 1. The bracket $[$,$] associated with \delta=D_{\theta}$ provides $\mathcal{V}(\mathcal{A}(1))$ with a Lie antialgebra structure isomorphic to the one denoted as $\mathcal{A K}(1)$ in [7]. 
The proposition is a consequence of a direct computation, the product of two elements of $\mathcal{V}(\mathcal{A}(1))$ satisfies

$$
\left[(u+\theta \phi) D_{\theta},(v+\theta \psi) D_{\theta}\right]=\left(u \psi+v \phi+\theta\left(u v^{\prime}-v u^{\prime}+2 \phi \psi\right)\right) D_{\theta} .
$$

On using suitable trigonometrical bases we recover formulas of [7, p.4] .

It is now natural to study the case when odd dimension $N$ is arbitrary: to any odd derivation $\delta$ of a superalgebra $\mathcal{A}$ is associated a graded algebra denoted as $\mathcal{V}(\mathcal{A}, \delta)$. We no longer have any Lie antialgebra structure if $N>2$, on the other hand

Proposition 2. The even part $\mathcal{V}(\mathcal{A}, \delta)_{0}$ is a Jordan algebra, and if furthermore $\delta^{2}=0$ the odd part $\mathcal{V}(\mathcal{A}, \delta)_{1}$ is a Jordan module.

Jordan algebras were first considered by the theoretical physicist Pascual Jordan (1902-1980) who used them to find the most suitable mathematical formalism in quantum mechanics. We shall not discuss here Jordan algebras in details, let us simply describe the standard example: take the algebra of square matrices, and symmetrize the product, you get a commutative but non associative algebra. For basic results on Jordan algebras, cf. [6].

For our proof, the following definition will be sufficient: a commutative algebra with a product "." is a Jordan algebra if for every $x, y$, one has

$$
(x \cdot y) \cdot(x \cdot x)=x \cdot(y \cdot(x \cdot x)) .
$$

(Jordan identity) Let us first compute with even elements of type $a \delta$ where $|a|=1$. In this case the product is commutative: $a \delta . b \delta=(a \delta b+b \delta a) \delta$. One finds

$$
\begin{gathered}
{[a \delta, a \delta]=2 a \delta a \delta} \\
{[b \delta,[a \delta, a \delta]]=\left(2 a \delta a \delta b+2 b \delta a \delta a+2 a b \delta^{2} a\right) \delta} \\
{[a \delta,[b \delta,[a \delta, a \delta]]]=(6 a \delta a \delta b \delta b) \delta+(2 b \delta a \delta a \delta a) \delta .}
\end{gathered}
$$

In particular terms in $\delta^{2}$ cancel miraculously. Besides, $[a \delta, b \delta]=(a \delta b+b \delta a) \delta$, $[[a \delta, a \delta][a \delta, b \delta]]=(6 a \delta a \delta b \delta b) \delta+(2 b \delta a \delta a \delta a) \delta$.

So one has $[[a \delta, a \delta][a \delta, b \delta]]=[a \delta,[b \delta,[a \delta, a \delta]]]$, which prove the first part of the proposition.

For the sequel, let us say first what Jordan modules are: we say that $M$ is a module on Jordan algebra $A$ if there are left and right actions such that $a . m=m . a$ for any $a \in A$ and $m \in M$, and such that space $A+M$ with the multiplication $(a, m) \cdot(b, n)=(a . b, a . n+m . b)$ be a Jordan algebra. 
If one developes and check formulas $J_{1}$ for $A+M$, one obtains two independent conditions

$$
\begin{aligned}
& \left.J_{2}\right)[a \delta, a \delta] \cdot(a \delta . n \delta)=a \delta \cdot([a \delta, a \delta] \cdot n \delta) \\
& \left.J_{3}\right)[a \delta, a \delta] .(b \delta . m \delta)+2[a \delta, b \delta] \cdot(a \delta . m \delta)=[b \delta,[a \delta, a \delta]] \cdot m \delta+2 a \delta .(b \delta .(a \delta . m \delta))
\end{aligned}
$$

Everything can be worked out explicitly, taking care of parities: $|a|=|\delta n|=1$ $|n|=|\delta a|=0$. One finds similarly

$$
\begin{aligned}
{[a \delta, a \delta] .(a \delta . n \delta)=(6 a \delta a \delta a \delta n} & +2 n \delta a \delta a \delta a) \delta=a \delta .([a \delta, a \delta] . n \delta) \\
{[a \delta, a \delta] .(b \delta . m \delta)+2[a \delta, b \delta] .(a \delta . m \delta) } & =12 a \delta a \delta a \delta n+6 b \delta m \delta a \delta a+6 m \delta a \delta a \delta b \\
& =[b \delta,[a \delta, a \delta]] . m \delta+2 a \delta .(b \delta .(a \delta . m \delta)) .
\end{aligned}
$$

For $\delta^{2} \neq 0$, as for example for $\delta=D_{\theta_{i}}=\theta_{i} \frac{\partial}{\partial t}-\frac{\partial}{\partial \theta_{i}}$ on $\mathcal{A}(N)$ the algebra of functions on the supercircle $S^{1 \mid N}$ in variables $t, \theta_{i}, i=1 \ldots . N$ the proof fails.

Conjecture: For $\delta^{2}=0$, the graded commutative algebra $\mathcal{V}(\mathcal{A}, \delta)$ is a Jordan superalgebra (cf. [6] and [5] for definitions).

\section{About Odd Derivations}

\subsection{Derivations of (Super) Symplectic Structures}

There exists two different kind of supersymplectic structures according to parity of the form

- orthosymplectic or even supersymplectic structures. For canonical coordinates $p_{i}, q_{i}, \theta_{j}, i=1 \ldots . n, j=1 \ldots N$ on a $2 n \mid N$-dimensional manifold, the form reads as $\omega=\sum_{i=1}^{n} \mathrm{~d} q_{i} \wedge \mathrm{d} p_{i}+\sum_{j=1}^{N} \frac{1}{2} \mathrm{~d} \theta_{j}^{2}$, and the corresponding Poisson bracket satisfies

$$
\{f, g\}=\sum_{i=1}^{n}\left(\frac{\partial f}{\partial p_{i}} \frac{\partial g}{\partial q_{i}}-\frac{\partial f}{\partial q_{i}} \frac{\partial g}{\partial p_{i}}\right)+\sum_{j=1}^{N} \frac{\partial f}{\delta \theta_{j}} \frac{\partial g}{\delta \theta_{j}} .
$$

- periplectic or odd symplectic structures on $N \mid N$-dimensional manifolds, with coordinates $x_{i}, \theta_{i}, i=1 \ldots . n$ for which the form is $\omega=\sum_{i=1}^{N} \mathrm{~d} x_{i} \wedge \mathrm{d} \theta_{i}$ while Poisson bracket reads as

$$
\{f, g\}=\sum_{i=1}^{N}\left(\frac{\partial f}{\partial x_{i}} \frac{\partial g}{\partial \theta_{i}}-\frac{\partial f}{\partial x_{i}} \frac{\partial g}{\partial \theta_{i}}\right) .
$$


Adjoint action of $a \in \mathcal{A}$ for those respective Poisson brackets defines an odd derivation of $\mathcal{A}$, if $a$ is odd (orthosymplectic case), or even (periplectic case).

\subsection{Sketch of Classification for $N=2$}

Let us consider the algebra $\mathcal{A}(2)$ of functions on the supercircle $S^{1 \mid 2}$ with variables $\left(t, \theta_{1}, \theta_{2}\right)$, and find an odd operator $\mathcal{D}=A \partial_{t}+U_{1} \partial_{\theta_{1}}+U_{2} \partial_{\theta_{2}}$ such that $\mathcal{D}^{2}=0$ (so that $A$ is odd, and $U_{1}, U_{2}$ even). One deduces three differential equations in $A, U_{1}, U_{2}$, it gives six equations in six unknown functions on $S^{1}$, and one easily gets the general form of the solution

$$
\mathcal{D}=\lambda\left(u_{2} \theta_{1}-u_{1} \theta_{2}\right) \partial_{t}+\left(u_{1}+\lambda u_{1}^{\prime} \theta_{1} \theta_{2}\right) \partial_{\theta_{1}}+\left(u_{2}+\lambda u_{2}^{\prime} \theta_{1} \theta_{2}\right) \partial_{\theta_{2}}
$$

with $\lambda$ being an arbitrary scalar, and $u_{1}, u_{2}$ being arbitrary functions in $t$.

\subsection{Supergeometric Interpretation}

One can think of an associative supercommutative algebra as the algebra of functions on a supermanifold, so an odd (even) derivation is an odd (even) vector field on the supermanifold; the condition $\delta^{2}=0$ is then nothing but the "master equation", essential in BV-quantization, $[Q, Q]=0$, as in [1]. See [10] for a survey on BV-quantization.

\section{In Way of Motivation}

The algebraic structure of infinite dimensional integrable systems theory is extensively developed in [3]. The well known KP hierarchies are obtained through construction of square root of stationary Schrödinger type operators $\partial_{x}^{2}+u(x)$ in one space dimension, after a detailed study of the algebra of pseudodifferential symbols in $\partial_{x}$. But what can we do with Schrödinger type operators when non stationary, or more generally when dimension is greater than one?

This type of operator has been studied from infinite dimensional Poisson geometry point of view for a particular case in [11], and slightly extended in the book [12], using Schrödinger -Virasoro symmetries. Also a different group-theoretical approach of that kind of operators is considered in [8].

An appropriate construction of Miura transform (cf. again [3] for definition of Miura transform in the case $d=1$ is given in [9], using a supergeometric framework, thanks to the "square root of time" $D_{\theta}^{2}=\frac{\partial}{\partial t}$ ). In order to generalize the 
computations, one has first to understand the structure of the algebra of pseudodifferential symbols generalized to supergeometric case, hence the considerations developed in this article.

\section{References}

[1] Alexandrov M., Schwarz A., Zaboronsky O. and Kontsevich M., The Geometry of the Master Equation and Topological Quantum Field Theory, International Journal of Modern Physics A. Particles and Fields. Gravitation Cosmology, Nuclear Physics 12 (1997) 1405-1429.

[2] Deligne P. and Morgan J., Notes on Supersymmetry (Following J. Bernstein), Quantum Fields and Strings: A Course for Mathematicians, vols. $1 \& 2$, Amer. Math. Soc., Providence 1999.

[3] Dickey L., Soliton Equations and Hamiltonian Systems, $2^{\text {nd }}$ Edn, World Scientific, River Edge 2003.

[4] Guieu L. and Roger C., L'algèbre et le groupe de Virasoro, Aspects géométriques et algébriques, généralisations, With an Appendix by Vlad Sergiescu, CRM, Montreal 2007.

[5] Kac V., Martinez C. and Zelmanov E., Graded Simple Jordan Superalgebras of Growth One, Memoirs of the American Mathematical Society 150, Providence 2001.

[6] McCrimmon K., A Taste of Jordan Algebras, Springer, New York 2004.

[7] Ovsienko V., Lie Antialgebras: Prémices, J. Algebra 325 (2011) 216-247.

[8] Ovsienko V. and Roger C., Looped Cotangent Virasoro Algebra and NonLinear Integrable Systems in Dimension 2+1, Comm. Math. Phys. 273 (2007) 357-378.

[9] Roger C., A Matrix Miura Transform, J. Geom. Phys. 74 (2013) 87-90.

[10] Roger C., Gerstenhaber and Batalin-Vilkovisky Algebras: Algebraic, Geometric, and Physical Aspects, Archivum Mathematicum 45 (2009) 301-324.

[11] Roger C. and Unterberger J., A Hamiltonian Action of the SchrödingerVirasoro Algebra on a Space of Periodic Time-Dependent Schrödinger Operators in (1 + 1)-Dimensions, J. Nonlinear Math. Phys. 17 (2010) 257-279. 
[12] Unterberger J. and Roger C., The Schrödinger-Virasoro Algebra, Mathematical Structure and Dynamical Schrödinger Symmetries, With a Foreword by Malte Henkel, Theoretical and Mathematical Physics, Springer, Heidelberg 2012.

Received 30 July 2015

Claude Roger

Institut Camille Jordan

Laboratoire associé au CNRS UMR 5208

Ecole Centrale de Lyon

INSA de Lyon, Université Lyon I

43 boulevard du 11 novembre 1918

F-69622 VILLEURBANNE Cedex, FRANCE

E-mail address: roger@math. univ-lyon 1 . fr 